\title{
Curing kinetics and mechanical properties of a composite hydrogel for the replacement of the nucleus pulposus
}

\author{
A.C. Borges ${ }^{a}$, P.-E. Bourban ${ }^{a}$, D.P. Pioletti ${ }^{b}$, J.-A.E. Månson ${ }^{a, *}$ \\ a Laboratoire de Technologie des Composites et Polymères (LTC), Ecole Polytechnique Fédérale de Lausanne (EPFL), Station 12, CH-1015 Lausanne, Switzerland \\ ${ }^{\mathrm{b}}$ Laboratoire de Biomécanique en Orthopédie (LBO), Ecole Polytechnique Fédérale de Lausanne (EPFL), CH-1015 Lausanne, Switzerland
}

\section{A R T I C L E I N F O}

Article history:

Available online 25 July 2010

\section{Keywords:}

A. Polymers

A. Nano composites

B. Curing

C. Elastic properties

D. Rheology

\begin{abstract}
A B S T R A C T
A polymer material system has been developed to propose an injectable, UV and in situ curable hydrogel with properties similar to the native nucleus pulposus of intervertebral disc. Neat hydrogels based on Tween $^{\circledR} 20$ trimethacrylates (T3) and N-vinyl-2-pyrrolidone (NVP) and composite hydrogels of same composition reinforced by nano-fibrillated cellulose were synthesized with different T3 concentrations and their curing kinetics was investigated by photorheology using UV light. The T3 concentration has an influence on the time of curing and final shear stiffness of the material. NFC does not alter the time of curing but increases the final mechanical performance of the hydrogels for a same chemical composition. Hydrogel samples, neat and composite, were then tested in unconfined compression at different hydration stages and in confined compression and their elastic modulus was determined. The amount of fluid present in the network is mostly responsible for the mechanical properties and NFC fibres proved to be an efficient reinforcement. The elastic modulus ranged from 0.02 to $8 \mathrm{MPa}$. Biocompatibility studies showed that cells are confluent at $90 \%$ and do not show any morphology change when in contact with the hydrogel. The present hydrogel can therefore be considered for NP replacement.
\end{abstract}

(c) 2010 Elsevier Ltd. All rights reserved.

\section{Introduction}

Lower back pain is mostly due to intervertebral disc (IVD) degeneration and in particular to nucleus pulposus (NP) degeneration [1]. About $80 \%$ of the world population will suffer from back pain $[2,3]$ and $75 \%$ of the cases will be a consequence of degenerative processes of the disc. Total disc arthroplasty [4,5] and NP replacement are two non-fusion techniques emerging as solutions to this issue. The ideal benefits of a non-fusion solution are the removal of the source of pain and the preservation of the biomechanical balancing and motion of the spine [4,5]. A classical approach to replacing the NP involves mimicking the structure and function of the native NP. The NP is described as a gel-like structure containing randomly disposed collagen fibres and radial elastin fibres [6]. The NP is inextensible, extremely deformable and very hydrophilic [7]. The mechanical properties in unconfined compression and viscoelastic properties in shear have been previously reported $[8,9]$, as well as mechanical requirements for a NP replacement [10].

The choice and shape of materials has evolved with the knowledge of disc mechanics. At the beginning, the focus was on the replacement of the NP by a material that could restore disc height

\footnotetext{
* Corresponding author. Tel.: +41 2169342 85; fax: +41 216935880

E-mail address: jan-anders.manson@epfl.ch (J.-A.E. Månson).
}

and preserve motion. The first attempt came in the late 1950s and early 1960s, with Ferstrom replacing the NP by stainless steel ball bearings. Hamby and Glaser tried PMMA and Nachemson tried silicon but none of these techniques gained acceptance at that time [11]. In 1973, [11] an injectable mixed silicon Dacron (PET fibres) was used but reported bone resorption and aberrant bone formation. After these poor results, efforts were focused on improved knowledge of the disc and its functions. The aim was still nucleus replacement but taking into account biomechanics and biology, thus preventing further degeneration. In 1981, new developments [11] suggested choosing the material not only for its ability to mimic kinematic motion but also for its viscoelastic properties, i.e., the fluid inflow and outflow. Hydrogels are close in properties to many living tissues and are defined as hydrophilic polymer networks which may absorb from $10 \%$ to $20 \%$ up to thousands of times their dry weight in water [12]. Owing to their unique properties such as biocompatibility, penetrability, sorption, flexible methods of synthesis, stimulus-responsive properties and elasticity, they have been used in a variety of applications from tissue engineering [9] as scaffolds, barriers, drug delivery systems, cell encapsulation, artificial muscles, biosensors, actuators, contact lens-based ophthalmic systems to microfluidics [12]. Carl et al. [11] and Goins et al. [13] reviewed several hydrogel implants being currently under investigation for the replacement of the NP. They can be divided in two categories: preformed and in situ curing implants. 
In situ curing NP replacements can be injected non-invasively through a small needle and therefore reduce the risk of expulsion when compared to preformed implants. None of these proposed hydrogel implants gained universal acceptance up to now.

Hydrogels produced by photopolymerization have been extensively investigated as biomaterials [14]. Photopolymerization is a method of choice since it involves relatively short reaction times and minimal heating, and it does not require organic solvents [15]. A key advantage of photopolymerized hydrogels over chemically or physically crosslinked hydrogels is that their mechanical properties and swelling behaviour may be tuned relatively easily by varying the molecular mass and/or the concentration of the precursors. Hydrogels in general have typically been used in applications where mechanical properties are not determinant [16]. To overcome this issue, composite hydrogels are the best alternative, resulting in materials which could maintain their shape, have suitable morphology, mechanical properties, high hydrophilicity and biocompatibility. Actually, there are many promising ways to create composite hydrogels: processing of copolymer hydrogels, of interpenetrating networks (IPNs) or of polymer hydrogels containing an inorganic phase. These components are introduced in the hydrogels either to modify their properties such as compatibility with biological tissues, mechanical properties and thermo and $\mathrm{pH}$ - responsivity, or to create new properties such as magnetic characteristics and antibacterial properties [17-20]. A hydrogel reinforced with natural biocompatible nanofibres seems to be the best choice for mimicking the human NP as the latter also contains fibres. Nanofibres used for the reinforcement need to increase the mechanical properties of the hydrogel without hindering dramatically its swelling ability. In this context, these fibres should possess a high water retention value, which is the case of nano-fibrillated cellulose (NFC). NFC is usually obtained after a series of mechanical disintegrations of the raw material [21]. Thanks to its high surface area, NFC has amazing water retention values up to $400 \%$ [22]. NFC has already been used as reinforcement in several applications. Zimmermann et al. [23] observed significant reinforcing effect of cellulose fibrils with diameters below $100 \mathrm{~nm}$ in polyvinyl alcohol (PVA) composites and hydroxypropyl cellulose composites.

The objective of this study was to investigate the curing kinetics, mechanical behaviour in uniaxial compression and biocompatibility of Tween ${ }^{\circledR} 20$ trimethacrylate (T3) and N-vinyl2-pyrrolidone (NVP) hydrogel blends reinforced with NFC as potential UV, in situ curing NP replacement.

\section{Experimental details}

\subsection{Preparation of samples}

The samples were composed of Tween ${ }^{\circledR} 20$ trimethacrylate (T3), synthesized in the laboratory from poly(oxyethylene 20 sorbitan) monolaurate (Tween ${ }^{\circledR} 20$ ), as crosslinker, N-vinyl-2-pyrrolidone (NVP), water and initiator Irgacure 2959 (I2959, Ciba). NVP was used as purchased from Sigma-Aldrich, Switzerland and I2959 was used as an aqueous solution ( $0.05 \mathrm{wt} . \%$ in water). The volume of initiator and water in these tests remained constant at $10 \mathrm{vol} . \%$ and $40 \mathrm{vol} . \%$ respectively and the concentrations of T3 and NVP changed. NFC fibres were provided by the Swiss Federal Laboratories for Materials Testing and Research (EMPA, Dübendorf, Switzerland) in a form of a gel of $8 \mathrm{wt} . \%$ of fibres in water. The diameter of the fibres varied from 2 to $100 \mathrm{~nm}$ and their length was higher than $1 \mu \mathrm{m}$. The gel was then diluted in water to yield a gel of 2 wt.\% of fibres, using a high-shear mixer, an ultra-turrax (IKA T25 digital, SN 25 10G).

\subsection{Evolution of kinetics by photorheology}

The evolution of the network was followed by photorheology on a controlled strain dynamic rotational rheometer (ARES, Rheomet- rics Scientific), coupled with a UV light source. The solutions were tested between two parallel plates of $25 \mathrm{~mm}$ in diameter. The upper plate was a quartz plate through which the UV light could reach the sample. A sample of $200 \mu \mathrm{l}$ was loaded on the lower plate and the gap was set to $0.3 \mathrm{~mm}$ in order to avoid evaporation during the test. A scanning of strain and frequency was made before crosslinking to determine the conditions of the tests. It was observed that at $15 \%$ of strain and with a frequency of $10 \mathrm{~Hz}$ at room temperature the material was in its linear viscoelastic range. The evolution of the storage modulus $G^{\prime}$, loss modulus $G^{\prime \prime}$, complex viscosity $\eta *$ and $\tan \delta$ were measured for $30 \mathrm{~min}$ for samples with a T3 concentration of 1 vol.\% (T3-1), 4.5 vol.\% (T3-4.5), 8 vol.\% (T3-8), 11.5 vol.\% (T311.5 ) and $15 \mathrm{vol} . \%$ (T3-15), adding $0.8 \mathrm{wt} \%$ of NFC fibres for the composite hydrogels. Triplicate experiments showed excellent reproducibility. The UV source used for photorheology was an EXFO Omnicure S2000. The UV intensity used was $15 \mathrm{~mW} / \mathrm{cm}^{2}$ and was measured using the Sola-Check (Solatell, UK). The UV intensity varied by less than $10 \%$ between two illuminations. For simplicity, only $G^{\prime}$ curves will be shown.

\subsection{Mechanical performance of hydrogels}

\subsubsection{Unconfined compression}

A well-mixed solution of T3, NVP, I2959 and water was used to cast samples of $2 \mathrm{~cm}$ in diameter and $5 \mathrm{~mm}$ high in silicon moulds resistant to UV light. Samples were exposed for $30 \mathrm{~min}$ to UV light with an intensity of $140 \mathrm{~mW} / \mathrm{cm}^{2}$ measured between 270 and $370 \mathrm{~nm}$ (Sola-Check, Solatell, UK).The samples were then redimensioned with a punch to cylinders of $8 \mathrm{~mm}$ of diameter and $5 \mathrm{~mm}$ high and tested in compression directly after polymerization, after drying at $100{ }^{\circ} \mathrm{C}$ for $24 \mathrm{~h}$ and after $24 \mathrm{~h}$ of rehydration in phosphate buffered saline (PBS) at $37^{\circ} \mathrm{C}$. Two concentrations of T3 were tested: 8 vol.\% (T3-8) and 15 vol.\% (T3-15). For the composite hydrogels, $0.8 \mathrm{wt} \%$ of NFC fibres were added to the samples before polymerization and dispersed by using an ultra-turrax (IKA T25 digital, SN 25 10G).

The stiffness of the hydrogel was determined in compression with DMA measurements. The samples were loaded between two parallel plates of $1 \mathrm{~cm}$ in diameter, enclosed in a furnace for isothermal measurements. The measuring device was a dynamic mechanical analyzer (DMA Q800, TA Instruments). The lower plate was fixed and the upper plate compressed the samples. A force ramp of $3 \mathrm{~N} / \mathrm{min}$ was applied until the force reached a maximum of $15 \mathrm{~N}$. For the rehydrated samples, the compression tests were made in immersion mode, in a PBS solution. The elastic modulus $E$ was calculated from the stress-strain curves between $0 \%$ and $10 \%$ of strain because the compressive strain of a healthy nucleus pulposus ranges from $-10 \%$ to $10 \%$ [24]. All tests were triplicated, showing a good reproducibility.

\subsubsection{Confined compression}

Neat hydrogel samples containing 4.5 vol.\% of T3 (T3-4.5 0 NFC) and composite hydrogel samples containing $4.5 \mathrm{vol} . \%$ of $\mathrm{T} 3$ and $0.4 \mathrm{wt} . \%$ of NFC (T3-4.5 0.4 NFC), prepared as mentioned in the previous section, at swelling equilibrium, were tested in compression in a confined environment on a UTS Z010, Zwick (Ulm, Germany). All tests were made in an immersion tank with PBS at $37^{\circ} \mathrm{C}$. The confinement device is shown in Fig. 1; it is composed of a centering plate inserted in the immersion tank and a plunger. Hydrogel samples were confined in a rigid silicon rubber ring and two metallic porous plates, placed on the centering plate, and the cylindrical plunger was compressed upon the hydrogel at a strain rate of $1 \mathrm{~mm} / \mathrm{min}$ until fracture. A preload of $10 \mathrm{mN}$ was applied. The design of the set-up ensures the centering of the samples, uniaxial compression and possible fluid flow during the tests. Three samples per measurements were performed and the values were aver- 


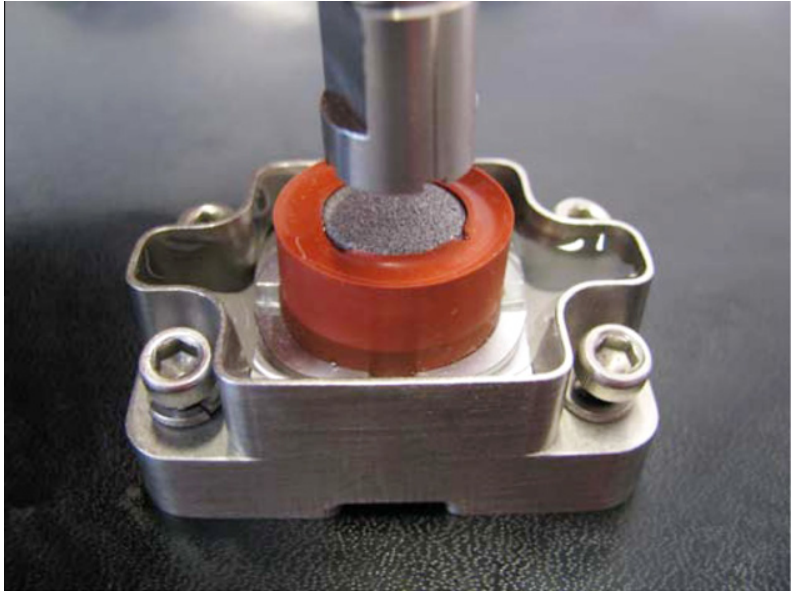

Fig. 1. Experimental set-up for confined compression showing the centering plate in the immersion tank, the silicon membrane and the porous plates enclosing the hydrogel sample allowing fluid flow.

aged. Confined compression modulus $E_{\text {conf }}$ was calculated between $14 \%$ and $15 \%$ of strain using a linear fit.

\subsection{Biocompatibility of hydrogels}

Cylinders of hydrogel ( $1 \mathrm{~cm}$ diameter and $5 \mathrm{~mm}$ height) kept in PBS were sterilized in an autoclave and then placed in Petri dishes. The affinity and morphology of different cell types toward the hydrogel were evaluated by a Giemsa coloration. Cell types used were articular cartilage foetal cells, intercostal cartilage foetal cells and bone foetal cells. The cells were seeded around the previously autoclaved hydrogel sample and let grow until reaching a confluence of about $90 \%$. Then the cells were fixed by methanol and coloured.

\section{Results and discussion}

\subsection{Curing kinetics}

Reaction kinetics is studied in situ. The aqueous solutions of hydrogels are loaded between two parallel plates which will built into the as-prepared gels a certain amount of stress. A time-sweep measurement is carried out for each different sample and the effect of T3 concentration is studied. During the time-sweep measurement, the storage modulus $\left(G^{\prime}\right)$, loss modulus $\left(G^{\prime \prime}\right)$, $\tan \delta$ and complex viscosity $\eta *$ are monitored, as shown in Fig. 2 for a T3-8 hydrogel and the reaction kinetics can be qualitatively evaluated. During the initial stage of the reaction, $G^{\prime}, G^{\prime \prime}, \tan \delta$ and $\eta *$ increase very fast indicating a growth of the chain size and network formation. In the intermediate stage, which occurs here in the first minutes of the reaction, $G^{\prime}$ increases faster and exceeds $G^{\prime \prime}$, while $\tan \delta$ decreases to a value near zero. This increase of $G^{\prime}$ is characteristic of the transformation of a Newtonian viscous fluid to an elastic solid due to network formation [25]. During the last stage of the reaction, $G^{\prime}$ continues to increase due to gel aging, while $G^{\prime \prime}$ is almost invariant and less than $G^{\prime}$, showing the dominant elasticity of the system and indicating the existence of a permanent three-dimensional network. An inflexion can be observed in these curves that is probably due to chain rearrangements and the formation of new chemical links enhanced by the shear stress applied to the sample. The more rapid increase of the storage modulus is characteristic of the sol-gel transition [25] and the gel time observed when $G^{\prime}$ crosses $G^{\prime \prime}$ is of paramount importance for the understanding of network formation and morphology of the future material.

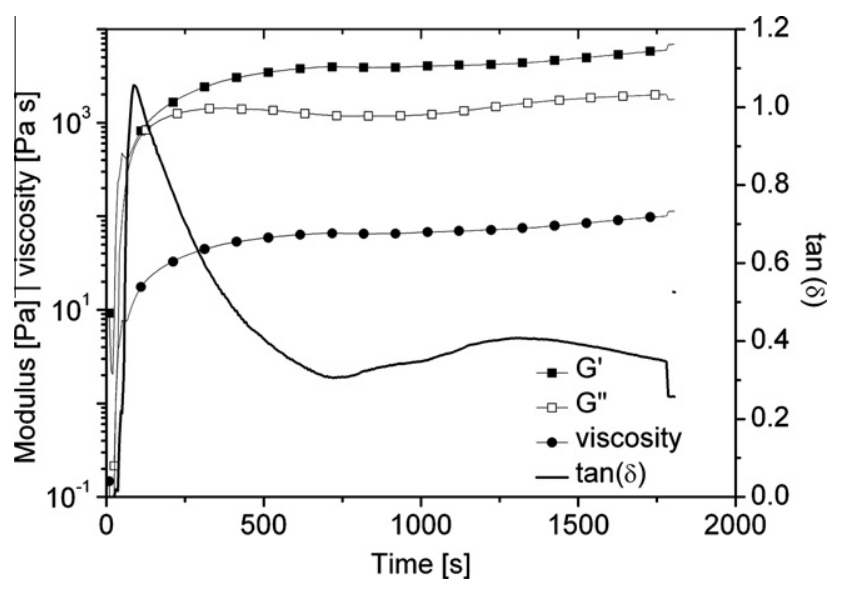

Fig. 2. Kinetics behaviour of a T3-8 hydrogel. The storage modulus $\left(G^{\prime}\right)$, loss modulus $\left(G^{\prime \prime}\right)$, tan $\delta$ and complex viscosity $\eta *$ are monitored as a function of irradiation time. The UV intensity was $15 \mathrm{~mW} / \mathrm{cm}^{2}$ and the measurements were done at room temperature (RT), with $15 \%$ of strain and at $10 \mathrm{~Hz}$.

All hydrogels present the same features and therefore, for clarity, only $G^{\prime}$ will be shown.

The concentration of T3 has an influence on the time needed for the polymerization and on the final values of $G^{\prime}$. Fig. 3 shows that at very small concentrations of $\mathrm{T} 3$, the plateau is not reached, which means that the polymerization is not complete. A small concentration of $\mathrm{T} 3$ means less reactive species in the solution. Therefore, when all the T3 molecules are crosslinked, the NVP molecules are not able to form the network as fast as before because of their lower reactivity. Reactivity ratios found for NVP in the literature range from 0.02 to 0.10 whereas reactivity ratios for methacrylates are around three, depending on the structure of the molecule $[15,26]$. This difference has shown to cause methacrylates to polymerize faster than NVP in solution polymerization of these polymers [27]. Increasing the T3 concentration also increases the polymerization rate. At a concentration of $4.5 \%$ and $8 \%$ in volume of T3, the time needed for polymerization is around $400 \mathrm{~s}$ whereas with concentrations of $11.5 \mathrm{vol} \%$ and $15 \mathrm{vol} \%$ the time of polymerization is around $700 \mathrm{~s}$. A fast polymerization is a key requirement for this application. The reason of this difference is explained by the step mechanisms of free radical crosslinking. After the initiation, the chain growth starts with the radicals attacking other molecules and forming the network. Once the network is formed, the

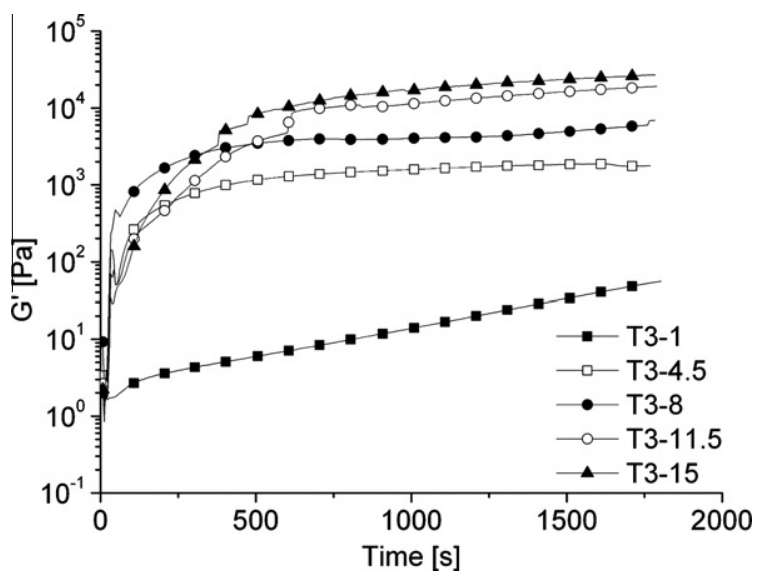

Fig. 3. Storage modulus $G^{\prime}$ of a T3/NVP hydrogel measured as a function of irradiation time for different T3 concentrations at RT and at $15 \mathrm{~mW} / \mathrm{cm}^{2}$ and with 10 vol.\% of initiator ( $15 \%$ strain, $10 \mathrm{~Hz}$ ). 
mobility of the still free molecules is decreased and the crosslinking becomes diffusion-controlled. The last molecules have difficulties in moving in the network and in combining with other molecules and the rate of reaction decreases.

The final values of the moduli and viscosity are presented in Table 1 . As the amount of T3 is increased, the shear stiffness and the viscosity of the final network increase as well. $G^{\prime}$ is always higher than $G^{\prime \prime}$, indicating that elastic links between the molecules have been formed. The viscous component represented by $G^{\prime \prime}$ is also important in the final hydrogels as the values are high. The final plateaus illustrate the formation of a stable 3-D network during polymerization. The difference in final values between T3-8 and T3-11.5 is more important than for T3-11.5 and T3-15. The diffusion-controlled step in the reaction mechanism is responsible for this phenomenon and hinders the final formation of chemical links in an already strongly crosslinked network. Therefore, there is probably a threshold value of T3 in the network, above which the time of curing and final properties are only dependent on the controlled-diffusion mechanism and for which the final mechanical properties cannot be significantly increased. Only concentrations of T3 of 4.5 vol.\% and above should be considered for this application because of short curing times and complete polymerization.

The T3-8 hydrogel reinforced by NFC shows the same kinetics behaviour as neat gels of T3 and NVP. The storage modulus $G^{\prime}$ was higher than the loss modulus $G^{\prime \prime}$ and the viscosity followed a similar trend by increasing and then reaching a plateau when the polymerization was complete (Fig. 4). $\tan \delta$ reaches a minimum at the beginning of the reaction and then increases as the network forms before reaching a plateau at the very end of the polymerization. The evolution of moduli of neat and reinforced gels is presented in Fig. 5. Initial values of moduli of composite hydrogels are significantly high and increase during polymerization. The dispersion of NFC in the T3-8 solution greatly increases the viscosity and before polymerization, the mixture has the characteristics of

Table 1

Final values of $G^{\prime}, G^{\prime \prime}$ and $\eta *$ as a function of T3 concentration.

\begin{tabular}{llll}
\hline & $G^{\prime}(\mathrm{Pa})$ & $G^{\prime \prime}(\mathrm{Pa})$ & $\eta *($ Pa.s $)$ \\
\hline T3-1 & 56.3 & 93.1 & 1.7 \\
T3-4.5 & $1757 \pm 82$ & $424 \pm 52$ & $31 \pm 1$ \\
T3-8 & $4786 \pm 702$ & $1876 \pm 282$ & $81 \pm 11$ \\
T3-11.5 & $15,253 \pm 2293$ & $7634 \pm 198$ & $272 \pm 31$ \\
T3-15 & $22,226 \pm 2878$ & $14,359 \pm 486$ & $423 \pm 34$ \\
\hline
\end{tabular}

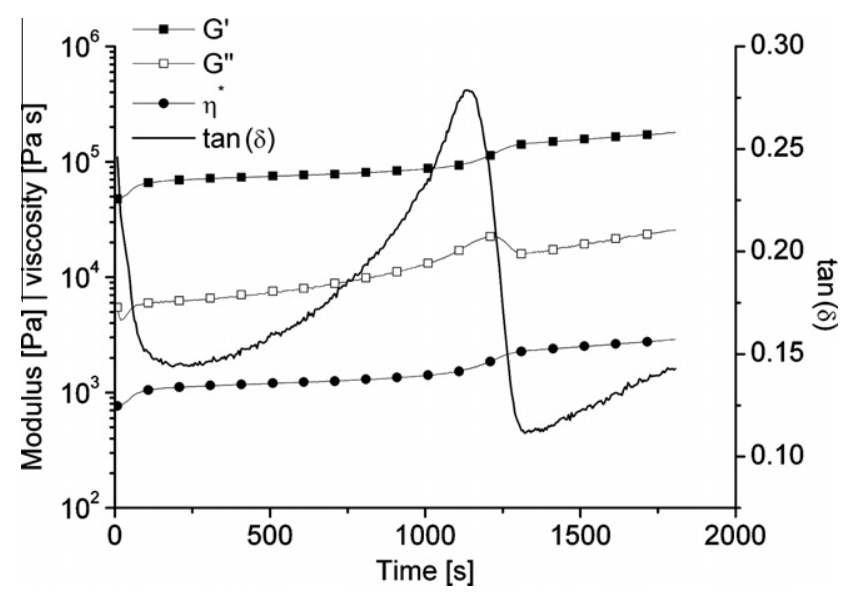

Fig. 4. Modulus of a T3-8/NVP and 0.8 wt.\% NFC gel measured as a function of irradiation time at RT and at $15 \mathrm{~mW} / \mathrm{cm}^{2}$ and with 10 vol.\% of initiator. Measurements were made with a strain of $0.5 \%$ and at a frequency of $10 \mathrm{~Hz}$.

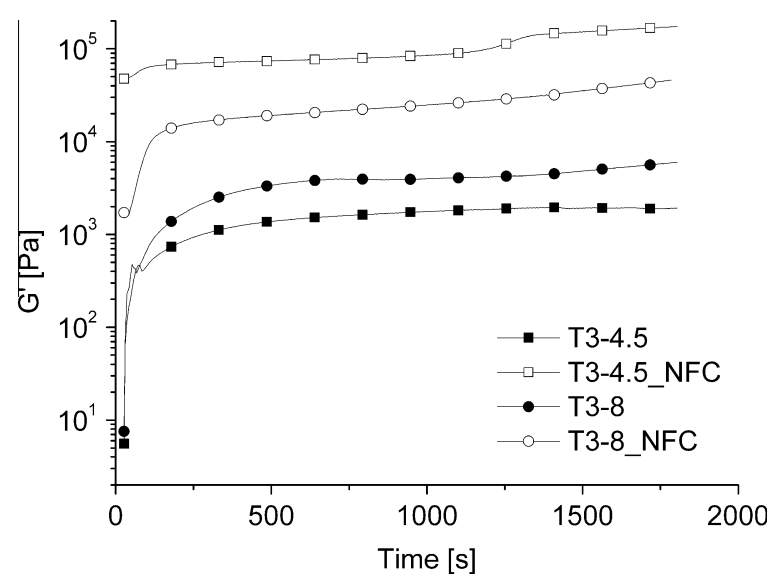

Fig. 5. Storage modulus $G^{\prime}$ of a T3/NVP and $0.8 \mathrm{wt} . \%$ NFC hydrogel measured as a function of irradiation time for different T3 concentrations at RT and at $15 \mathrm{~mW} / \mathrm{cm}^{2}$ and with 10 vol.\% of initiator (15\% strain, $10 \mathrm{~Hz}$ ).

a gel. Therefore, there is no gel point and the plateau is reached very fast. It is also interesting to notice that the time of polymerization did not change for both systems, indicating that NFC fibres do not interfere in the network formation. The mechanism of formation of the network could then be compared to the formation of an interpenetrating network (IPN), where the T3/NVP hydrogel network is formed around the NFC network. The final storage modulus $G^{\prime}$ and viscosity for the reinforced hydrogel are higher than for the neat hydrogel, showing the reinforcement effect of NFC. The hydrogel containing a lower amount of T3 benefits more of the reinforcement effect of NFC as it can be observed in Fig. 5. For higher concentrations of $\mathrm{T} 3$, the radical diffusion in the forming network is hindered by the NFC network, resulting in a greater amount of dangling chains and cyclization. There is therefore a threshold concentration of T3 above which the addition of NFC does not influence the shear stiffness of the network. It can also be noticed that there is only a slight increase in $G^{\prime}$ and viscosity for the T3-4.5 sample once the UV irradiation starts, whereas for the T3-8 sample, both values increase by one order of magnitude before reaching the plateau. This indicates that for less crosslinked networks, the mechanical properties are determined by the NFC amount only. Table 2 summarizes the final values obtained for $G^{\prime}, G^{\prime \prime}$ and $\tan \delta$ for neat and reinforced hydrogels of composition T3-4.5 and T3-8. The increase of the values is very significant, ranging up to several orders of magnitude. It can be noticed that $G^{\prime}$ is always higher than $G^{\prime \prime}$, indicating an elastic behaviour of the composite hydrogels. With higher initial viscosity that could be an advantage for the application, to avoid leakage of the material in situ prior to polymerization, and curing times similar to neat hydrogels and finally higher shear moduli, composite hydrogels should be considered for the replacement of the NP.

\subsection{Unconfined compression}

The mechanical behaviour of two types of hydrogels was investigated at different conditions (dried, after polymerization and

Table 2

Final values of $G^{\prime}, G^{\prime \prime}$ and $\eta *$ for neat and reinforced hydrogels.

\begin{tabular}{lccc}
\hline & $G^{\prime}(\mathrm{Pa})$ & $G^{\prime \prime}(\mathrm{Pa})$ & $\eta *(\mathrm{~Pa} \mathrm{~s})$ \\
\hline T3-4.5 & $1757 \pm 82$ & $424 \pm 52$ & $31 \pm 1$ \\
T3-4.5_NFC & $1.4 \cdot 10^{5} \pm 31,000$ & $19,463 \pm 3251$ & $2159 \pm 477$ \\
T3-8 & $4786 \pm 702$ & $1876 \pm 282$ & $81 \pm 11$ \\
T3-8_NFC & $31,857 \pm 6198$ & $6750 \pm 198$ & $540 \pm 102$ \\
\hline
\end{tabular}


after rehydration) as shown in Figs. 6 and 7. Three different samples were tested for each condition and a good reproducibility of the measurements was observed. For both hydrogels, the dried samples showed an elastic behaviour and they were much more resistant than the samples after polymerization and rehydrated. As they were vacuum dried for 24 h before testing, the polymerization had time to complete and the evaporation of water allowed the network to shrink, thus reinforcing it. They showed no viscous behaviour. The samples after polymerization showed that the fluid present in the network is responsible for the mechanical behaviour of the material. Until about 30\% of deformation, the curves were linear, showing a good elastic response and after that value, an exponential trend was observed due to a rearrangement of the polymer chains as a response to the applied stress. The barreling effect of the hydrogel cylinders when compressed could also contribute to the exponential trend. The rehydrated samples at swelling equilibrium showed lower network stiffness when compressed. Here again, the fluid present in the network is responsible for this phenomenon. The drying step and then the increase of fluid in the network did not alter dramatically its properties. For the T3-8 hydrogel, the rehydrated samples plateaued at about $40 \%$ of strain corresponding to a destruction of the sample and the same was observed for T3-15 rehydrated samples.

In Fig. 8, the two hydrogel systems were compared and it can be observed that the concentration of $\mathrm{T} 3$ does not influence significantly the mechanical properties. The values of stress vs strain

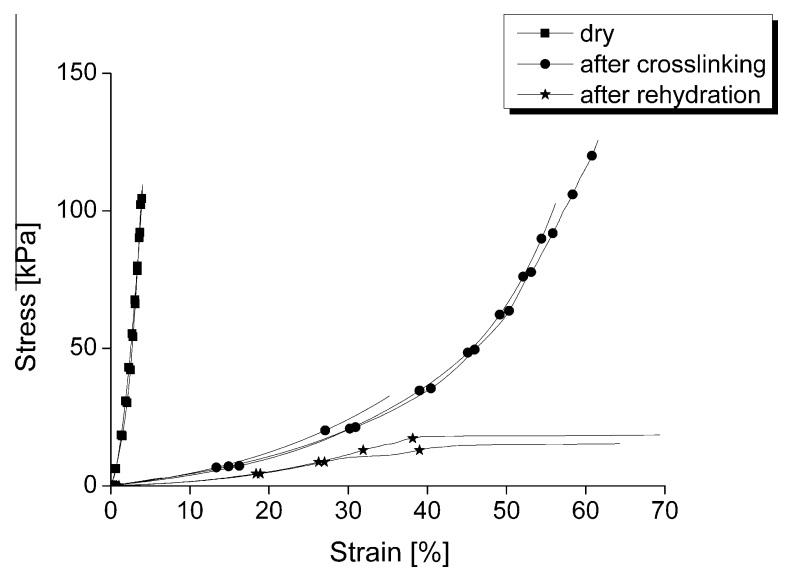

Fig. 6. Stress-strain curve for a T3-8 hydrogel at three different conditions. The force ramp was $3 \mathrm{~N} / \mathrm{min}$ until reaching $15 \mathrm{~N}$.

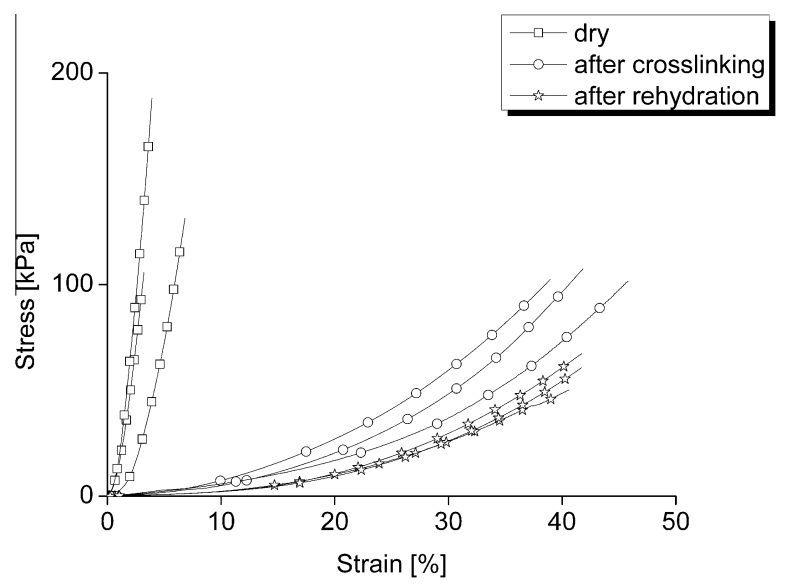

Fig. 7. Stress-strain curve for a T3-15 hydrogel at three different conditions. The force ramp was $3 \mathrm{~N} / \mathrm{min}$ until reaching $15 \mathrm{~N}$.

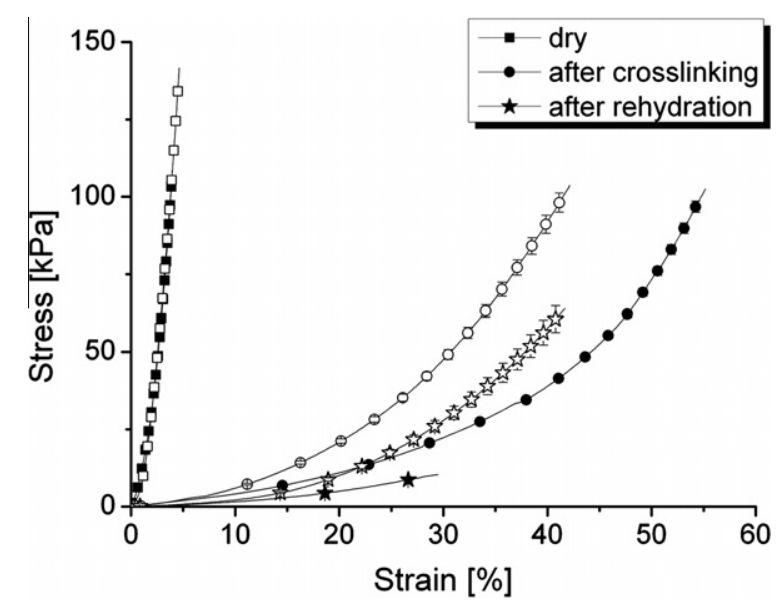

Fig. 8. Comparison of the mechanical behaviour in compression of two different hydrogels, T3-8 (solid symbols) and T3-15 (hollow symbols) at here different hydration degrees. The force ramp was $3 \mathrm{~N} / \mathrm{min}$ until reaching $15 \mathrm{~N}$.

for dried samples are very similar even if the concentration of crosslinker is increased. However, when the network is hydrated, the mechanical properties change completely for both concentrations of T3 and the amount of T3 plays a role on the obtained results. A more crosslinked network will absorb less fluid and therefore have a greater stiffness. The elastic modulus $E$ of the different samples at different conditions was calculated from the slope of the curves (Table 3 ). The value of $E$ for the native nucleus pulposus is in the range of 3-6 $\mathrm{kPa}$ [8]. Dried hydrogels are too stiff for the application. The values of the modulus for the hydrated hydrogels are in the range of the values of the native NP and these hydrogels could be considered for NP replacement. Reinforced T3-8 and T3-15 hydrogels were tested in compression after polymerization, after drying and after rehydration for $24 \mathrm{~h}$ at $37^{\circ} \mathrm{C}$ in PBS. The reinforced hydrogels presented the same behaviour as the neat hydrogels with very stiff behaviour when dried and becoming softer with an increasing amount of fluid. Fig. 9 illustrates this behaviour. Dried samples of reinforced hydrogel showed the same elastic behaviour as samples of neat hydrogel. Their mechanical properties are greatly increased due to the lack of fluid and the shrinking of the network. The reinforced samples tested just after polymerization did not show a significant exponential behaviour characteristic of the stiffening of the material due to chain rearrangement and release of water during compression. There is a small inflexion at the beginning of the curve, probably due to minimal rearrangements of the network and release of peripheral PBS. After what, fluid is trapped in the very intricate network and the material behaves as an elastic solid without any influence due to fluid diffusion in the network. When the samples were dried, the water was evaporated and during the rehydration, fluid penetrated in the network. As mentioned by Turbak et al. [28], NFC fibres lose their mechanical and wettable properties once dried. This phenomenon could be observed here as the rehydrated samples of reinforced hydrogel had mechanical properties comparable to the T3-8 hydrogels that have weaker networks. Except this

Table 3

Elasticity modulus $E$ of hydrogels with two different concentrations of T3.

\begin{tabular}{llll}
\hline & $\begin{array}{l}E \text { (dried samples) } \\
(\mathrm{MPa})\end{array}$ & $\begin{array}{l}E \text { (after } \\
\text { polymerization) } \\
(\mathrm{MPa})\end{array}$ & $\begin{array}{l}E \text { (after } \\
\text { rehydration) }(\mathrm{MPa})\end{array}$ \\
\hline T3-8 & 2.2 & 0.05 & 0.03 \\
$\mathrm{~T} 3-15$ & 2.5 & 0.08 & 0.04 \\
\hline
\end{tabular}




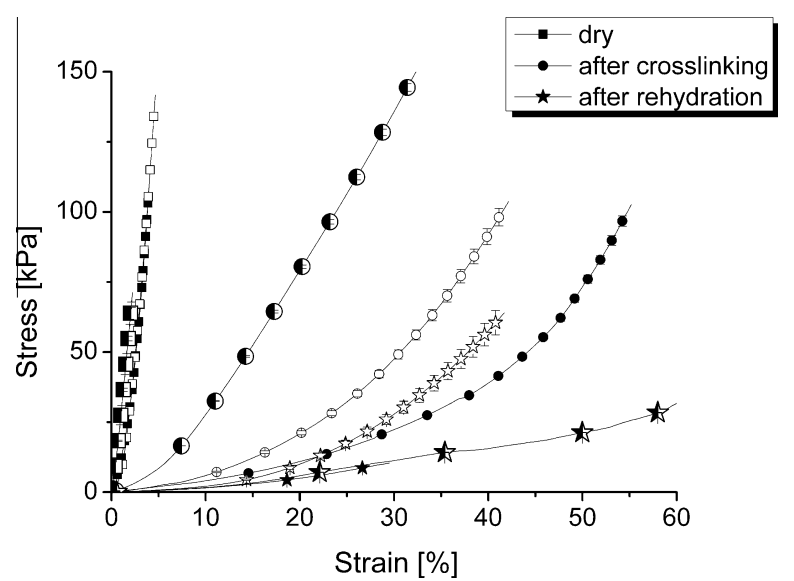

Fig. 9. Stress-strain curve for a T3-8 (solid symbols), T3-15 (hollow symbols) and T3-8_NFC (solid and hollow symbols) hydrogel at three different conditions. The force ramp was $3 \mathrm{~N} / \mathrm{min}$ until reaching $15 \mathrm{~N}$.

particular case, T3-8 hydrogels with NFC showed better mechanical properties in static compression due to the interpenetrating network formed. The elastic modulus $E$ was calculated again here between $0 \%$ and $10 \%$ of strain and the values are presented in Table 4 . The reinforcement of the network by NFC was clearly proved. For dried samples, the modulus is increased four times and for samples after polymerization, the modulus was one order of magnitude higher than for a neat hydrogel. Hydrated neat and composite hydrogels showed nevertheless mechanical properties in compression close to the native NP [8] and can be considered as potential candidates for NP replacement. Further tests are needed to show the reinforcement effect of NFC in fully hydrated hydrogel networks.

\subsection{Confined compression}

In the spine, the NP is confined by the annulus fibrosus (AF). In order to test the hydrogels in conditions approaching the real biological use, a set-up was developed to perform compression tests in an immersed confined environment. This is not strict confinement because fluid flow is possible through the porous plates that enclose the hydrogel. The silicone membrane was chosen to be very stiff compared to the hydrogel, in order to avoid barreling and other measurement artefacts. Only two hydrogels were tested in confined compression: the neat hydrogel T3-4.5 and the hydrogel containing 0.4 wt.\% of NFC, T3-4.5 0.4 NFC. Typical stress-strain curves of confined hydrogels in compression are presented in Fig. 10. These curves do not show the real mechanical behaviour of the hydrogels but mainly the hydrostatic pressure applied to the silicone membrane. The first part of the curves, before the inflexion point, represents the behaviour of the hydrogel until it is completely confined. The evolution of the stress-strain curve until $8-10 \%$ of strain is similar to the evolution observed with unconfined compression. At the inflexion point, the hydrogels become almost incompressible with a Poisson's ratio of about 0.5 , as reported in literature for incompressible materials [9]. After the inflexion point, only the hydrostatic pressure transmitted to the

Table 4

Elasticity modulus $E$ for neat and composite hydrogels.

\begin{tabular}{llll}
\hline & $\begin{array}{l}E \text { (dried } \\
\text { samples) }(\mathrm{MPa})\end{array}$ & $\begin{array}{l}E \text { (after } \\
\text { polymerization) } \\
(\mathrm{MPa})\end{array}$ & $\begin{array}{l}E \text { (after } \\
\text { rehydration) } \\
(\mathrm{MPa})\end{array}$ \\
\hline T3-8 & 2.2 & 0.05 & 0.03 \\
T3-8_NFC & 8 & 0.2 & 0.02 \\
\hline
\end{tabular}

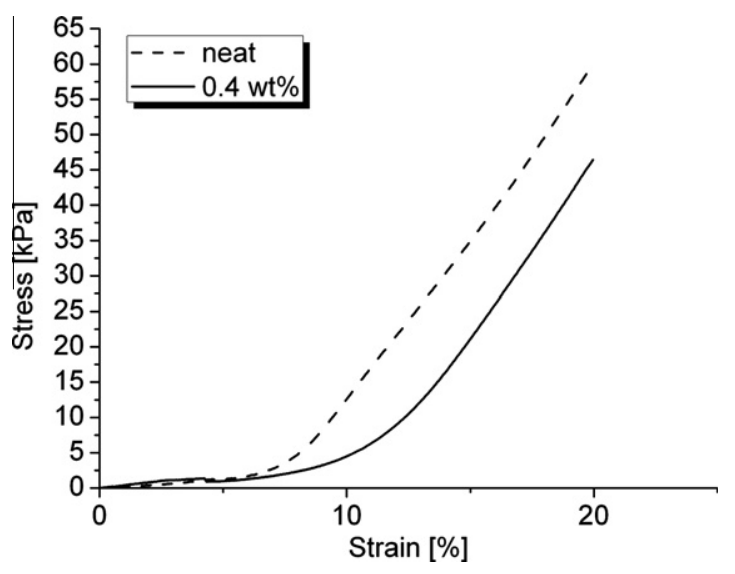

Fig. 10. Stress-strain curves of a T3-4.5 and T3-4.5 hydrogel with 0.4 wt.\% of NFC in confined compression.

membrane is measured. The inflexion point does not appear at the same strain for each tested hydrogel: the appearance of the incompressible nature of the hydrogel depends on the amount of PBS in its network. With a higher quantity of PBS in the network, the incompressible nature will appear at lower strains. The hydrogel without NFC (neat) is the one which swells the most. The curve of the latter is therefore shifted towards highest stress values given that the hydrogel becomes incompressible at a earlier stage. The hydrogel with the less PBS in its network (T3-4.5 0.4 NFC) will consequently have the inflexion point at higher strains. The elastic modulus $E_{\text {conf }}$ was calculated from the linear part of the curves between $14 \%$ and $15 \%$ of strain by a linear interpolation. The results are presented on the Fig. 11. Considering the standard deviation, the elastic modulus $E_{\text {conf }}$ does not vary significantly when NFC are added to the network as it was observed in unconfined compression of hydrogels. The measured values for the moduli are therefore the values of the stiffness of the confinement silicone membrane because of the hydrostatic pressure transmitted by the hydrogel to the membrane and not the stiffness of the material. Therefore, the confinement of such a material should be investigated further by changing the set-up and particularly the thickness of silicone membrane. Other limitations include the friction of the piston, the porous plates and the hydrogel against the silicone membrane. Nevertheless, this study demonstrated the potential of the developed hydrogels to transmit loads to the surrounding annulus fibrosus.

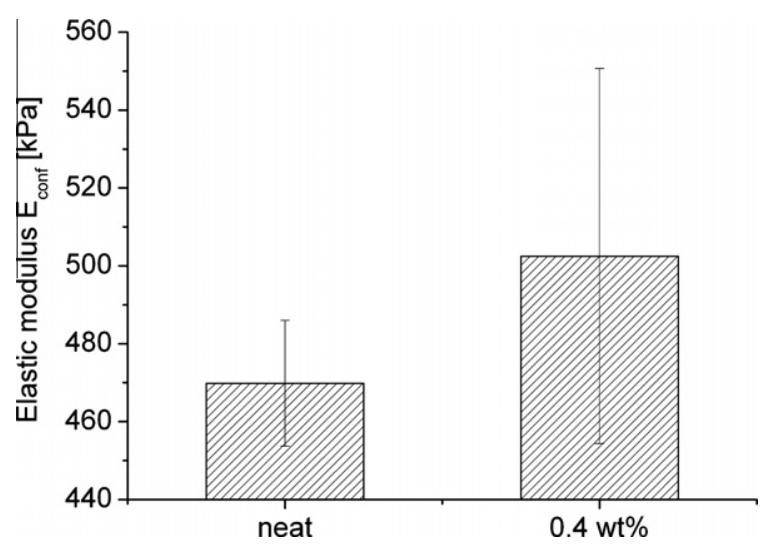

Fig. 11. Elastic modulus $E_{\text {conf }}$ in confined compression for a T3-4.5 and T3-4.5 hydrogel with $0.4 \mathrm{wt}$.\% of NFC. 

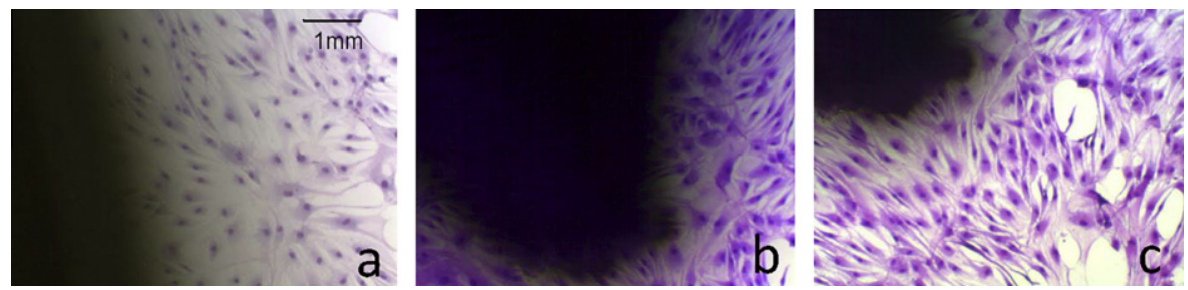

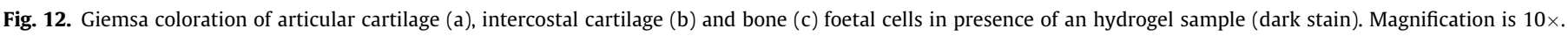

\subsection{Biocompatibility studies}

Foetal cells seeded around the hydrogels were used as preliminary assessment to determine the biocompatibility of the material. Cells were disposed in a Petri dish without touching the hydrogel sample and after some days, the confluence of cells reached $90 \%$, surrounding the hydrogel completely. Cells were then fixed by methanol and coloured by the Giemsa solution. Only cells attached to the Petri dish could be fixed and coloured indicating that only living cells are shown in Fig. 12. The cells do not present any granulation or anomalous morphology indicating that the hydrogel or any non-crosslinked monomer has an effect on the cell's metabolism. Further tests are being performed to investigate the effect of the material on cell growth.

\section{Conclusions}

The curing kinetics of hydrogels composed of Tween ${ }^{\circledR} 20$ trimethacrylate (T3) and N-vinyl-2-pyrrolidone (NVP) has been investigated by photorheology. The curing time and final shear modulus of freshly polymerized hydrogels were influenced by the amount of T3 in the network. NFC proved to have a reinforcement effect in shear, property that could be very important for the design of a NP replacement.

Stress-strain curves of the hydrogels tested in unconfined compression illustrate the influence of the fluid content on their mechanical performance, the elastic modulus ranging from 0.03 to $2.5 \mathrm{MPa}$ for hydrogels at different moisture contents. NFC showed a reinforcement effect for hydrogels of same chemical composition. The native NP has elastic modulus values ranging from 3 to $6 \mathrm{kPa}$ [8]. Fully hydrated composite hydrogels could be potential candidates for NP replacement with elastic modulus around $20 \mathrm{kPa}$. Confined compression of neat and composite hydrogels showed the capacity of the material to transmit the loads to the surrounding structures like the native NP. Preliminary biocompatibility tests showed good affinity of cells towards the hydrogel.

Swelling studies are currently being performed on neat and reinforced hydrogels to determine the fluid uptake rate of hydrogels and its influence on mechanical properties.

\section{Acknowledgements}

Financial support was provided from Swiss National Science Foundation. We thank Dr. Christopher J. Plummer for valuable discussions and Mr. M. Oggier and Ms. S. Jaccoud for their help. We also thank Dr. Constantin Schizas for helpful discussions.

\section{References}

[1] Urban JPG, Roberts S. Degeneration of the intervertebral disc. Arthritis Res Therapy 2003;5(3):120-30.

[2] Anderson DG, Tannoury C. Molecular pathogenic factors in symptomatic disc degeneration. Spine J 2005;5(Suppl. 6. 1):S260-6.
[3] Antoniou J, Steffen T, Nelson F, Winterbottom N, Hollander AP, Poole RA, et al. The human lumbar intervertebral disc: evidence for changes in the biosynthesis and denaturation of the extracellular matrix with growth, maturation, ageing, and degeneration. J Clin Invest 1996;98(4):996-1003.

[4] Bono CM, Garfin SR. History and evolution of disc replacement. Spine J 2004;4(Suppl. 6):145S-50S.

[5] Lee CK, Goel VK. Artificial disc prosthesis: design concepts and criteria. Spine J 2004;4(Suppl. 6):209S-18S.

[6] Yu J, Winlove PC, Roberts S, Urban JP. Elastic fibre organization in the intervertebral discs of the bovine tail. J Anat 2002;201(6):465-75.

[7] Rannou F, Mayoux-Benhamou MA, Poiraudeau S, Revel M. Anatomy, biology, physiology, and biomechanics of intervertebral disk and other anatomical structures of the lumbar spine [Disque intervertébral et structures voisines de la colonne lombaire: Anatomie, biologie, physiologie et biomécanique]. EMCRhumatologie-Orthopedie. 2004;1(6):487-507.

[8] Cloyd JM, Malhotra NR, Weng L, Chen W, Mauck RL, Elliott DM. Material properties in unconfined compression of human nucleus pulposus, injectable hyaluronic acid-based hydrogels and tissue engineering scaffolds. Eur Spine J 2007;16(11):1892-8.

[9] Iatridis JC, Setton LA, Weidenbaum M, Mow VC. The viscoelastic behavior of the non-degenerate human lumbar nucleus pulposus in shear. J Biomech 1997;30(10):1005-13.

[10] Bao QB, Yuan HA. Prosthetic disc replacement: the future? Clin Orthop Related Res 2002(394):139-45

[11] Carl A, Ledet E, Yuan H, Sharan A. New developments in nucleus pulposus replacement technology. Spine J 2004;4(Suppl. 6):325S-9S.

[12] Hoffman AS. Hydrogels for biomedical applications. Adv Drug Deliv Rev 2002;54(1):3-12

[13] Goins ML, Wimberley DW, Yuan PS, Fitzhenry LN, Vaccaro AR. Nucleus pulposus replacement: an emerging technology. Spine J 2005;5(Suppl. 6):317S-24S.

[14] Peppas N. Hydrogels in medicine and pharmacy. CRC Press; 1986.

[15] Lee JH, Bucknall DG. Swelling behavior and network structure of hydrogels synthesized using controlled UV-initiated free radical polymerization. J Polym Sci, Part B: Polym Phys 2008;46(14):1450-62.

[16] Kopecek J. Hydrogels: from soft contact lenses and implants to self-assembled nanomaterials. J Polym Sci, Part A: Polym Chem 2009;47(22):5929-46.

[17] Bordi F, Paradossi G, Rinaldi C, Ruzicka B. Chemical and physical hydrogels: two case systems studied by quasi elastic light scattering. Physica A 2002;304(1-2):119-28.

[18] Haraguchi K, Farnworth R, Ohbayashi A, Takehisa T. Compositional effects on mechanical properties of nanocomposite hydrogels composed of poly(N, Ndimethylacrylamide) and clay. Macromolecules 2003;36(15):5732-41.

[19] Haraguchi K, Takehisa T. Nanocomposite hydrogels: a unique organicinorganic network structure with extraordinary mechanical, optical, and swelling/de-swelling properties. Adv Mater 2002;14(16):1120-4.

[20] Haraguchi K, Takehisa T, Fan S. Effects of clay content on the properties of nanocomposite hydrogels composed of poly(N-isopropylacrylamide) and clay. Macromolecules 2002;35(27):10162-71.

[21] Turbak AF, Snyder FW, Sandberg KR. Microfibrillated cellulose, a new cellulose product: properties, uses, and commercial potential. J Appl Polym Sci Appl Polym Sympos 1983;37:815-27.

[22] Lu J, Wang T, Drzal LT. Preparation and properties of microfibrillated cellulose polyvinyl alcohol composite materials. Compos Part A: Appl Sci and Manufact 2008;39(5):738-46.

[23] Zimmermann T, Pol̂hler E, Geiger T. Cellulose fibrils for polymer reinforcement. Adv Eng Mater 2004;6(9):754-61.

[24] Tsantrizos A, Ito K, Aebi M, Steffen T. Internal strains in healthy and degenerated lumbar intervertebral discs. Spine 2005;30(19):2129-37.

[25] Sarmento VHV, Frigério MR, Dahmouche K, Pulcinelli SH, Santilli CV. Evolution of rheological properties and local structure during gelation of siloxanepolymethylmethacrylate hybrid materials. J Sol-Gel Sci Technol 2006:37(3):179-84.

[26] Al-Issa MA, Davis TP, Huglin MB, Yip DCF. Copolymerizations involving Nvinyl-2-pyrrolidone. Polymer 1985;26(12):1869-74.

[27] Faragalla MM, Hill DJT, Whittaker AK. The copolymerization of N-vinyl-2pyrrolidone with 2-hydroxyethyl methacrylate. Polym Bull 2002;47(5):421-7.

[28] Turbak AF, Snyder FW, Sandberg KR. Microfibrillated cellulose, a new cellulose product: properties, uses, and commercial potential. J Appl Polym Sci. Appl Polym Sympos 1983;37:815-27. 